\title{
PROMOÇÃO DO ENVELHECIMENTO SAUDÁVEL DE RESIDENTES DE UMA INSTITUIÇÃO DE LONGA PERMANÊNCIA: RELATO DE EXPERIÊNCIA
}

HEALTHY AGING PROMOTION AMONG RESIDENTS OF A LONG-TERM CARE INSTITUTION: AN EXPERIENCE REPORT

PROMOCIÓN DEL ENVEJECIMIENTO SALUdABLE ENTRE RESIDENTES DE UNA INSTITUCIÓN DE CUIDAdOS A LARGO PLAZO: UN INFORME DE EXPERIENCIA

Marcela Meirelles Tozzi 1 Luis Henrique Ribeiro Barbosa ${ }^{2}$

Nathan Mendes Souza ${ }^{3}$

Fernanda Piana Santos Lima de Oliveira ${ }^{4}$

Palavras-chave: Saúde do Idoso; Instituição de Longa Permanência para Idosos; Envelhecimento Saudável; Promoção da Saúde; Terapias Complementares.

Keywords:

Elderly Health; Long-Term Care Institution; Healthy Aging; Health Promotion; Complementary Therapies.

Palabras clave:

Salud del Anciano; Institución de Cuidados a Largo Plazo; Envejecimiento Saludable; Promoción de la Salud; Terapias Complementarias.

Submetido: 17 de Set. de 2019

Aprovado: 25 de Nov. de 2020

Autor(a) para Correspondência: Marcela Meirelles Tozzi

Av. Prof. Alfredo Balena, 190 Santa Efigênia - Belo Horizonte, MG CEP:30130-100 E-mail:marcelamtozzi@gmail.com

\section{RESUMO}

Este artigo descreve a vivência de estudantes de medicina em intervenções promotoras do envelhecimento saudável em uma instituição de longa permanência (ILPI) para idosos em Minas Gerais. Trata-se de relato de experiência sobre intervenções para promoção da saúde e implementação de estratégias mais efetivas junto a idosas residentes em uma ILPI. Realizou-se um diagnóstico situacional por estimativa rápida, mediado pela observação e escuta, para levantar os principais problemas do serviço. As ações desenvolvidas envolveram grupos de musicoterapia, atividades cognitivas, caminhada e ioga/otago e ocorreram em 2017 e 2018. Constatou-se adesão das idosas às atividades propostas. Vale destacar que as atividades cognitivas proporcionaram aumento da capacidade de socialização. 0 trabalho em equipe e a sensibilidade para ouvir e cativar o outro, além da exposição a vulnerabilidades das mais diversas formas são algumas das reflexões proporcionadas por essa experiência. As atividades demonstraram potencial para desenvolver as habilidades sociais e motoras das idosas residentes na instituição em estudo, contribuindo para um envelhecimento mais ativo.

1. Aluna de Graduação em Medicina na Universidade Federal de Minas Gerais (UFMG).E-mail: marcelamtozzi@gmail. com ORCID: https://orcid.org/0000-0002-8699-8953

2. Aluno de Graduação em Medicina na UFMG. E-mail: luishrb96@gmail.com 0RCID: https://orcid.org/0000-0002$\underline{2256-0629}$

3. Mestre em Educação Médica pela Universidade Federal de Ouro Preto (UFOP). Professor na UFMG. Membro da Sociedade Brasileira de Medicina de Família e Comunidade (SBMFC). E-mail: nameso@gmail.com 0RCID: https:// orcid.org/0000-0002-4341-1964

4. Doutora em Odontologia pela UFMG. Professora no Centro Universitário FIPMOC (UNIFIPMoc). E-mail: fernandapiana@gmail.com 0RCID: https://orcid.org/0000-0002-8826-6852

Certificação de redação científica: E.L.Freire Editora. Edição de texto: Evandro L. Freire. Revisão de provas: Texto definitivo lido e validado pelos autores. 


\section{ABSTRACT}

This article describes the experience of medical students in healthy aging promotion interventions at a longterm care institution for the elderly (LTCIE) in Minas Gerais, Brazil. This is an experience report on interventions for health promotion and deployment of more effective strategies along with elderly women living in a LTCIE. A situational diagnosis was made by quick estimation, mediated by observation and listening, in order to raise the main problems in the service. The actions taken involved music therapy groups, cognitive activities, walking and yoga/'otago', and they took place in 2017 and 2018. It was found that the elderly women adhered to the proposed activities. It is worth noticing that cognitive activities led to increased socialization ability. Teamwork and sensitivity to listen and captivate others, in addition to exposure to much vulnerability are some of the reflections provided by this experience. The activities showed potential to develop social and motor skills of elderly women living in the institution under study, thus contributing to a more active aging.

\section{RESUMEN}

Este artículo describe la experiencia de estudiantes de medicina en intervenciones de promoción del envejecimiento saludable en una institución de cuidados a largo plazo para ancianos (ICLPA) en Minas Gerais, Brasil. Este es un informe de experiencia sobre intervenciones para promoción de la salud y el despliegue de estrategias más efectivas junto con ancianas que viven en una ICLPA. Se realizó un diagnóstico situacional mediante estimación rápida, mediada por la observación y la escucha, con el fin de plantear los principales problemas en el servicio. Las acciones realizadas involucraron grupos de musicoterapia, actividades cognitivas, caminata y yoga/"otago", y se llevaron a cabo en 2017 y 2018. Se constató que las ancianas adhirieron a las actividades propuestas. Cabe señalar que las actividades cognitivas condujeron a una mayor capacidad de socialización. El trabajo en equipo y la sensibilidad para escuchar y cautivar a los demás, además de la exposición a vulnerabilidades de las más diversas formas son algunas de las reflexiones que brinda esta experiencia. Las actividades mostraron potencial para desarrollar las habilidades sociales y motoras de las ancianas que viven en la institución en estudio, contribuyendo asi a un envejecimiento más activo.

\section{INTRODUÇÃ 0}

0 envelhecimento da população é notório no Brasil, que superou os 30,2 milhões de idosos em 2017 ${ }^{1}$. Com o aumento dessa parcela da população, as instituições de longa permanência para idosos (ILPI), também denominadas casas de repouso e clínicas geriátricas na literatura, passaram a ser mais requisitadas ${ }^{2}$. Além disso, reconhecem-se diversas questões que afetam esse perfil populacional, como situações de estresse devido a condições crônicas de saúde, distúrbios do sono, dor, perdas, além de transtornos mentais como depressão e Doença de Alzheimer, que, muitas vezes, somam-se à insuficiência de recursos pessoais e sociais ${ }^{3}$.

A Organização Pan-Americana de Saúde (OPAS) conceituou o envelhecimento como “um processo sequencial, irreversível, não patológico, de deterioração de um organismo maduro, de maneira que o tempo o torne menos capaz de fazer frente ao estresse do meio-ambiente e, portanto, aumente sua possibilidade de morte" ${ }^{14: 8}$. Dessa insuficiente adaptação surgem os problemas de saúde típicos do idoso: a) declínio cognitivo e neuropsicomotor; b) aumento da fragilidade e dependência; e c) maior suscetibilidade a doenças crônicas e agudas 5 .

Considerando o exposto, de fato, o envelhecimento é irreversivel, contudo, particularidades da vida o moldam desde a juventude, tornando-o único para cada pessoa ${ }^{6}$. Assim, o processo de desenvolvimento e manutenção da capacidade funcional viabiliza o bemestar em idade avançada e, com isso, um envelhecer saudável ${ }^{6-13}$. Este tem como fatores indicativos: a) baixo risco de doenças e de incapacidades funcionais; b) funcionamento mental e físico excelentes; e c) envolvimento ativo com a vida ${ }^{7}$.

Nesse sentido, exalta-se o lazer como algo imprescindivel para a manutenção da saúde e a autovalorização das pessoas na terceira idade; faz-se necessário que a população se convença de que não é só o trabalho que dá sentido à vida. Ademais, quando se participa de atividades de lazer, estabelecemse relações com o meio ambiente e o mundo, uma condição que favorece o inter-relacionamento das pessoas e o bem-estar ${ }^{8}$.

0 desenvolvimento de ações de lazer, como atividades físicas, musicais, artísticas, artesanais ou de outra ordem, ganham importância na diminuição 
do estresse, da depressão, da angústia e da ansiedade e no preenchimento do tempo ocioso, melhorando a qualidade de vida. Além disso, ganhos sociais e funcionais mantêm e promovem a independência e a autonomia daqueles que envelhecem ${ }^{9}$.

Considerando o exposto, o objetivo deste estudo foi:

- Descrever a vivência de estudantes de medicina em intervenções promotoras do envelhecimento saudável em uma ILPI em Minas Gerais.

\section{METODOLOGIA}

Trata-se de estudo do tipo relato de experiência resultante de vivência de estudantes do curso de medicina da Universidade Federal de Minas Gerais (UFMG). Essa vivência adveio da disciplina “Iniciação à Atenção Primária em Saúde" (IAPS), com inserções nos semestres 2017.1, 2017.2 e 2018.1, contabilizando 27 momentos.

A proposta interventiva foi posta em prática considerando o método simplificado do planejamento estratégico situacional ${ }^{10}$. Realizou-se diagnóstico situacional por estimativa rápida, mediado por observação e escuta, para levantar os principais problemas da ILPI - versando sobre questões que envolviam o envelhecimento saudável, o tema explorado pelos discentes.

Para tal vivência foram consideradas as necessidades das residentes, sendo as estratégias baseadas em evidências científicas para um envelhecimento saudável. Realizou-se um planejamento de atividades que complementasse as ações já adotadas na ILPI, trazendo inovações com vistas à melhoria da saúde das residentes. Adotouse, ainda, uma tipificação de grupos que considera as orientações do Ministério da Saúde (MS). Este sugere 3 grandes grupos para abordar as atividades mais prevalentes nas ILPI:

- 0 primeiro grupo deve estar relacionado ao acompanhamento terapêutico - incluindo musicoterapia e escuta de relatos, experiências, queixas e conversas abertas - e busca o aumento da interação social, com criação de vínculos e compartilhamento de histórias;

- 0 segundo grupo abrange prática corporal/ atividades físicas e terapêuticas, práticas esportivas e de lazer;

- E o terceiro grupo engloba as atividades socioculturais, como a confecção de objetos de arte, estimulando a criatividade e a coordenação motora e as atividades voltadas a jogos de conquista, que auxiliam o raciocínio lógico, a memória e a interação entre os idosos. Além dessas atividades, também se recomenda a organização de eventos culturais, como apresentações musicais e peças de teatro, com o propósito de beneficiar idosos com deficiência. Por fim temos a atividade estética, cujo objetivo é estimular o autocuidado e a higiene pessoal ${ }^{11,12}$.

Tendo em vista a importância das atividades em grupo, mostrou-se indispensável definir qual seria o tipo de atividade e o tema a propor. Essas escolhas devem ser discutidas com os participantes de modo que estejam mais adequadas às demandas e às realidades locais ${ }^{13}$, o que foi realizado pela equipe discente e docente da disciplina IAPS.

\section{RESULTADOS E DISCUSSÃO}

A vivência ocorreu em 27 momentos, distribuídos ao longo de 3 semestres. Em cada um deles ocorreram 9 encontros para a realização das atividades na ILPI. Outros 6 encontros foram realizados entre os acadêmicos e os professores para o planejamento das atividades, prática de oficinas sobre método clínico centrado na pessoa, habilidades de comunicação, educação em saúde, mudança de comportamento e devolutivas dos resultados obtidos na ILPI em conjunto com o centro de saúde. Os grupos de atividades foram os mesmos nos 3 semestres, sendo organizados de acordo com a metodologia proposta, com adesão das residentes em todas as atividades.

No planejamento das intervenções, a musicoterapia se deparou com um obstáculo: os tipos de músicas a utilizar nas atividades. A falta de meio padronizado de seleção de estímulos musicais já foi identificada em outro estudo como um dos principais problemas que levam ao mau aproveitamento dos benefícios da musicoterapia. Contudo, já havia sido levantado que a velocidade de processamento aumenta com músicas mais agitadas. Diante disso, as músicas

\section{...ganhos sociais $e$ funcionais mantêm e promovem a independência e a autonomia daqueles que envelhecem.}




\section{Utilizar jogos \\ e brincadeiras facilita \\ a aprendizagem e a prática \\ de comportamentos específicos.}

selecionadas foram da década de 1970, consideradas mais animadas e de maior interesse pelas moradoras. Quanto à velocidade de processamento, diante da falta de método para quantificar, observou-se que as residentes se recordavam das músicas que haviam sido tocadas na semana anterior, além das atividades realizadas ${ }^{14,15}$.

Durante a musicoterapia se estimulou: a) a escuta passiva dos estímulos sonoros; b) o ato de cantar; e c) a realização de movimentos de pequena amplitude para o desenvolvimento cognitivo. Apesar de um estudo mostrar que os efeitos a curto prazo da musicoterapia não melhoram a função cognitiva dos idosos, a realização de movimentos, mesmo aqueles de pequena amplitude, junto com a musicoterapia, resultou em maior adesão à atividade. A avaliação da possivel melhora cognitiva não pôde ser realizada por falta de instrumentos padronizados ${ }^{16,17}$.

Dentre as atividades cognitivas, a primeira foi "desenhos para colorir e roda de conversa". Segundo estudo, um componente fundamental para a promoção e manutenção da saúde de idosos institucionalizados é a instauração de interações significativas entre os residentes das instituições, o que pressupõe uma abordagem capaz de favorecer um trabalho voltado às práticas de linguagem (como as rodas de conversa $)^{18}$. Essa atividade consistia em levar uma folha contendo diversos desenhos a colorir e, em seguida, uma roda de conversa para estimular a discussão sobre a identificação das imagens e dos assuntos relacionados a ela. Todavia, devido às limitações motoras e de linguagem de algumas participantes, a atividade não pôde ser desenvolvida como previsto e serviu apenas como roda de conversa. Outros obstáculos para a atividade foram: a) desinteresse; b) falta de tempo; e c) doenças. Isso corrobora estudos que relatam os motivos da não adesão de idosas a grupos de convivência ${ }^{19}$.

A segunda atividade foi o bingo. Utilizar jogos e brincadeiras facilita a aprendizagem e a prática de comportamentos específicos ${ }^{20}$. Essa atividade proporcionou estímulo à memória, à audição, ao raciocínio, à compreensão de comandos e de número eà habilidade de contagem. Confirmando as percepções, a literatura cita o bingo como recurso positivo na promoção da saúde por estimular muitas áreas do aparelho cognitivo relacionadas à atenção, ao foco, à concentração e à memória imediata. Ademais, tal atividade envolve a percepção visual e auditiva, o movimento motor preciso e consiste em instrumento capaz de fornecer interação social e comunitária, além de meio para escapar do autoisolamento ${ }^{21}$.

Já a terceira atividade foi a chamada "Caixa de Pandora", que estimula a memória, a atenção, a linguagem, o reconhecimento de figuras e a socialização. Essa atividade consistia na passagem de uma sacola opaca contendo objetos da vida cotidiana. Cada participante retirava um objeto às cegas e dizia para a roda seu significado e sua utilização. Na segunda parte da atividade cada idosa precisava lembrar e listar todos os objetos que tinham sido retirados da sacola, testando sua atenção e sua memória. Vale ressaltar que intervenções complexas envolvendo técnicas de memorização, relaxamento e atenção podem gerar efeitos positivos e duradouros em idosos ${ }^{22}$, especialmente quando empregadas em grupo.

Em relação à caminhada, sabe-se que é o exercício aeróbico mais praticado por idosos. Pesquisas recentes apontaram que eles podem beneficiar-se dos exercícios aumentando não só sua resistência e sua força muscular, mas também seu equilíbrio e sua mobilidade. Isso pode reduzir os riscos de quedas e lesões, melhorando a autonomia funcional. Além disso, deve-se destacar os benefícios que abrangem as esferas biológica, psicológica e social, como: a) melhora da autoestima e da autoconfiança; b) diminuição da ansiedade e do estresse; e c) melhora do humor e da qualidade de vida ${ }^{23-25}$.

A caminhada foi proposta para as idosas da ILPI, independente de seu grau de dependência. Entretanto, houve pouca adesão das moradoras e a duração média das caminhadas foi de 15 minutos. As barreiras mais frequentes à prática relatadas pelas idosas foram: a) falta de tempo; b) medo de lesão; e c) necessidade de repouso. 0s trajetos escolhidos para a atividade foram decisivos para os resultados, em razão dos obstáculos no caminho, como passeios com degraus, desníveis e falta de conservação das ruas em geral.

Quanto às atividades de ioga/otago, devido ao 
número e à frequência dos encontros, a avaliação dos objetivos de aumento da força muscular, coordenação motora e redução do número de quedas é algo complexo. Os exercícios realizados somente uma vez por semana podem não ter contribuído de modo significativo para a evolução das idosas que participaram, uma vez que o material utilizado como referência preconiza que os exercícios devem ser praticados no mínimo 3 vezes por semana ${ }^{26}$. Entretanto, vale ressaltar que há outras ações na ILPI relacionadas à atividade física, como caminhada e fisioterapia, de modo que a prática de ioga/otago pode ter contribuído para os benefícios relatados.

Independente do alcance dos objetivos propostos, ioga/otago atraiu bastante participação e as sessões se tornaram oportunidades de descontração, despertando o poder lúdico da atividade física. Além disso, as sessões constituíram um espaço para o desenvolvimento psicossocial e afetivo, uma vez que as idosas usufruíram da escuta, da conversa e do toque físico para revelar aspectos sobre suas rotinas, seus gostos pessoais, suas histórias do passado e seus desafios atuais.

Diante disso, define-se que a capacidade física e o grau de dependência não são condicionados somente pela idade, mas por uma série de fatores como, por exemplo, o cuidado com a saúde durante a vida ${ }^{6}$. Isso pôde ser observado durante as atividades, visto que a idosa mais assídua e disposta do grupo era a de maior idade. Notou-se que as idosas com menor dependência eram as mais dispostas nas atividades. Isso gerou uma questão:

- As idosas têm menor disposição ao exercício por terem menor condicionamento físico ou têm menor condicionamento físico por terem menor disposição ao exercício?

Por fim, cabe citar o impacto que a experiência relatada trouxe aos alunos. Desenvolver atividades em um local com múltiplas vulnerabilidades e ser demandado a deixar um impacto positivo é um desafio que proporciona aprendizado. 0 trabalho

$$
\begin{gathered}
\text {...as sessões } \\
\text { constituíram um } \\
\text { espaço para } \\
\text { o desenvolvimento } \\
\text { psicossocial e } \\
\text { afetivo... }
\end{gathered}
$$

em equipe e a sensibilidade para ouvir e cativar o outro, além da exposição a vulnerabilidades das mais diversas formas são algumas das muitas reflexões que tal experiência proporcionou. 0 exercício das habilidades de comunicação e da empatia foram frutos desse projeto e isso se refletirá na prática médica diária por meio da construção de uma boa relação-médico paciente, além de um cuidado mais humanizado.

\section{CONCLUSÃO}

As atividades cognitivas influenciaram a capacidade de socialização, observada em idosas mais interativas a fim de todas as sessões. Essas atividades precisaram ocorrer de modo distinto de acordo com os diferentes graus de dependência das participantes, mostrando-se possivel garantir maior participação nas atividades em grupo e manter o pilar da socialização.

A caminhada foi a atividade com menor adesão e maiores desafios de execução. Considerando que há um tempo mínimo de prática estabelecido para a obtenção de benefícios da atividade e sabendo que as idosas participantes não faziam outras atividades aeróbicas, constatou-se insuficiência de tempo, de frequência e, provavelmente, de resultados obtidos em comparação com o que se recomenda na literatura. Entretanto, não foram aplicados exames e/ou questionários relativos à condição física antes e depois dos 27 encontros, o que implica dificuldade de avaliação.

Quanto à prática de ioga/otago, as idosas apreciaram os exercícios. Uma boa relação entre aplicadores e idosas se mostrou fundamental para a participação e a continuidade na atividade. Observou-se que a capacidade de realização correta dos exercícios propostos servia de reforço positivo, sendo o contrário também verdadeiro.

A orientação sobre os benefícios das atividades e a possibilidade de melhora do condicionamento físico com o tempo é importante. Assim, é recomendável aumentar a frequência das sessões semanais junto com a prescrição de exercícios que possam ser realizados autonomamente pelas idosas, criando uma rotina diária de exercícios.

As atividades relacionadas à musicoterapia tiveram alta adesão. Contudo, a avaliação de seus reais impactos não pôde ser realizada em função do tempo de realização, do não uso de instrumento de avaliação de benefícios e da falta de padronização 
dos tipos de estímulos sonoros adotados.

A avaliação dos impactos das intervenções foi uma limitação deste estudo, além de seu curto período. Todavia, este relato sugere a implementação ou o aperfeiçoamento de experiências semelhantes.

Por fim, os acadêmicos envolvidos se beneficiaram com a experiência de um contato humanizado e de seus efeitos na construção de uma boa relação interpessoal, além do uso de evidências científicas para embasar as ações em saúde na prática médica.

\section{CONTRIBUIÇÃO DOS AUTORES}

Marcela Meirelles Tozzi e Luis Henrique Ribeiro Barbosa contribuíram com a realização da pesquisa e a redação do manuscrito. Nathan Mendes Souza contribuiu com o delineamento do estudo e a revisão crítica do manuscrito. Fernanda Piana Santos Lima de Oliveira contribuiu com a realização da pesquisa, o delineamento do estudo e a redação e revisão crítica do manuscrito.

\section{REFERÊNCIAS}

1. Paradella R. Número de idosos cresce $18 \%$ em 5 anos e ultrapassa 30 milhões em 2017 [document on the internet]. 2018 [cited 2019 Feb 4]. Available from: https://agenciadenoticias.ibge. gov.br/agencia-noticias/2012-agencia-de-noticias/ noticias/20980-numero-de-idosos-cresce-18-em-5anos-e-ultrapassa-30-milhoes-em-2017

2. Camarano AA, Kanso S. As instituições de longa permanência para idosos no Brasil. Rev Bras Estud Popul [serial on the internet]. 2010 [cited 2020 Dec 4];27(1):232-5. Available from: https://www.scielo. br/pdf/rbepop/v27n1/14.pdf

3. Rabelo DF, Neri AL. Intervenções psicossociais com grupos de idosos. Rev Kairós [serial on the internet]. 2013 [cited 2020 Dec 4];16(4):43-63. Available from: https://revistas.pucsp.br/index. php/kairos/article/viewFile/20022/14897

4. Brasil. Envelhecimento e saúde da pessoa idosa [serial on the internet]. Brasília (DF): Ministério da Saúde; 2006 [cited 2017 Nov 7]. Available from: http://189.28.128.100/dab/docs/publicacoes/ cadernos ab/abcad19.pdf

5. Leonardo KC, Talmelli LFS, Diniz MA, Fhon JRS, Fabrício-Wehbe SCC, Rodrigues RAP. Avaliação do estado cognitivo e fragilidade em idosos mais velhos, residentes no domicílio. Ciênc Cuid Saúde [serial on the internet]. 2014 [cited 2020 Dec 4];13(1):1207. Available from: http://www.periodicos.uem.br/ ojs/index.php/CiencCuidSaude/article/view/20033/ pdf 121
6. Organização Mundial da Saúde. Resumo: Relatório Mundial de Envelhecimento e Saúde [document on the internet]. 2015 [cited 2017 Nov 7]. Available from: http://sbgg.org.br/wp-content/uploads/2015/10/ OMS-ENVELHECIMENT0-2015-port.pdf

7. Rowe JW, Kahn RL. Human aging: usual and successful. Science. 1987;237(4811):143-49.

8. Davim RMB, Dantas SMM, Lima VM, Lima JFV. 0 lazer diário como fator de qualidade de vida: o que pensa um grupo da terceira idade. Ciênc Cuid Saúde [serial on the internet]. 2008 [cited 2017 Nov 7];2(1):19-24. Available from: file:///D:/5563Texto \%20do\%20artigo-16747-1-10-20081023.pdf

9. Fleurí $A C P, A l m e i d a A C S$, Diniz $A J$, Magalhães $L A D$, Ferreira LHC, Prata MTM, et al. Atividades lúdicas com idosos institucionalizados. Enferm Rev [serial on the internet]. 2013 [cited 2017 Nov 7];16(1):507. Available from: file:///D:/13018-Texto $\% 20$ do $\% 20$ artigo-46690-1-10-20161001.pdf

10. Campos FCC, Faria HP, Santos MA. Planejamento e avaliação das ações de saúde [document on the internet]. Belo Horizonte: Universidade Federal de Minas Gerais; 2010 [cited 2019 Feb 4]. Available from: https://www.nescon.medicina.ufmg.br/ biblioteca/registro/Planejamento e avaliacao das acoes de saude $2 / 3$

11. Castro VC, Carreira L. Leisure activities and attitude of institutionalized elderly people: a basis for nursing practice. Rev Latinoam Enferm [serial on the internet]. 2015 [cited 2020 Dec 4];23(2):30714. Available from: https://www.scielo.br/pdf/rlae/ v23n2/0104-1169-rlae-23-02-00307.pdf

12. Chanda ML, Levitin DJ. The neurochemistry of music. Trends Cogn Sci. 2013;17(4):179-93.

13. Bottilori S, Rosi A, Russo R, Vecchi T, Cavallini E. The cognitive effects of listening to background music on older adults: processing speed improves with upbeat music, while memory seems to benefit from both upbeat and downbeat music. Frontiers in Aging Neuroscience. $2014 ;(6): 284$.

14. Machado ABM, Haretel LM. Neuroanatomia funcional. 3. ed. São Paulo: Atheneu; 2006.

15. Li HC, Wang HC, Chou FH, Chen KM. The effect of music therapy on cognitive functioning among older adults: a systematic review and meta-analysis. J Am Med Dir Assoc. 2015;16(1):71-7.

16. Andrade EL, Matsudo SMM, Matsudo VKR, Araújo $T L$, Andrade DR, Oliveira LC, et al. Barriers and motivational factors for physical activity adherence in elderly people in developing country. Med Sci Sports Exerc. 2000;33(7):141. 
17. Souza IAL, Massi G, Berberian AP, Guarinello $A C$, Carnevale L. The impact of discursive linguistic activities in promoting the health of elderly people in a long-term care institution. Audiology Commun Res. $2015 ; 20(2): 175-81$.

18. Santos IB, Lucy G, Matos NM, Vale MS, Santos FB, Cardenas CJ, et al. Oficinas de estimulação cognitiva adaptadas para idosos analfabetos com transtorno cognitivo leve. Rev Bras Enferm [serial on the internet]. 2012 [cited 2020 Dec 4];65(6):962-8. Available from: https://www.scielo.br/pdf/reben/ $\underline{v 65 n 6 / a 12 v 65 n 6 . p d f}$

19. Araujo LSA, Moreira ACA, Freitas CASL, Silva MAM, Do Val DR. Idosos e grupos de convivência: motivos para a não adesão. Sanare (Sobral, Online) [serial on the internet]. 2017;16(1):58-67. Available from: https://sanare.emnuvens.com.br/sanare/ article/view/1140/625

20. Sobel BP. Bingo vs. physical intervention in stimulating short-term cognition in Alzheimer's disease patients. Am J Alzheimers Dis 0ther Demen. $2001 ; 16(2): 115-20$.

21. Chariglone IPF, Janczura GA. Contribuições de um treino cognitivo para a memória de idosos institucionalizados. Psico USF [serial on the internet]. 2013 [cited 2020 Dec 4];18(1):13-22. Available from: https://www.scielo.br/pdf/pusf/ v18n1/v18n1a03.pdf

22. Coelho CS, Coelho IC. Comparação dos benefícios obtidos através da caminhada e da hidroginástica para a terceira idade. II Encontro de Educação Física e Áreas Afins Núcleo de Estudo e Pesquisa em Educação Física; 2007 0ct 26-27, Teresina, BR. Anais. Teresina (PI): Universidade Federal do Piauí; 2007.

23. Nakatani AYK, Silva LB, Bachion MM, Nunes DP. Capacidade funcional em idosos na comunidade e propostas de intervenções pela equipe de saúde. Rev Eletrônica Enferm [serial on the internet]. 2009 [cited 2019 Feb 4];11(1):144-50. Available from: https://revistas.ufg.br/fen/article/view/46899

24. Maciel MG. Atividade física e funcionalidade do idoso. Motriz Rev Educ Fís [serial on the internet]. 2010 [cited 2019 Feb 4];16(4):1024-32. Available from: https://www.scielo.br/pdf/motriz/v16n4/ a23v16n4.pdf

25. Junqueira LCFL, Centro de Telessaúde HC/UFMG. Treino prevenção de quedas [document on the internet]. Belo Horizonte: Universidade Federal de Minas Gerais; 2017 [cited 2019 Feb 4]. Available from: https://ares.unasus.gov.br/acervo/handle/ ARES/8801

26. Irigaray TQ, Schneider RH, Gomes I. Efeitos de um Treino Cognitivo na Qualidade de Vida e no Bem-
Estar Psicológico de Idosos. Psicol Reflex Crít [serial on the internet]. 2011 [cited 2020 Dec 4];24(4):8108. Available from: https://www.scielo.br/pdf/prc/ v24n4/a22v24n4.pdf

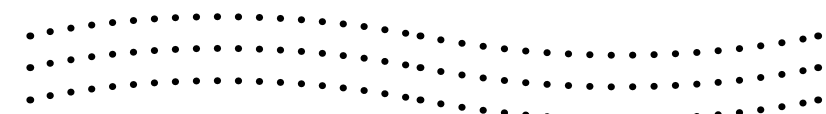

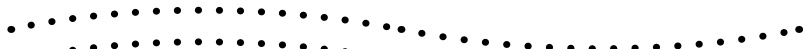
$\ldots \ldots \ldots \ldots \ldots \ldots \ldots \ldots \ldots \ldots \ldots \ldots \ldots \ldots \ldots \ldots \ldots \ldots$

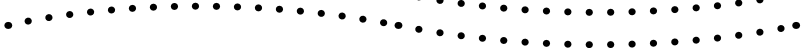

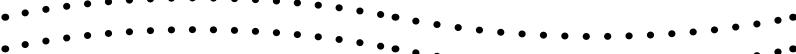
$\ldots \cdots \cdots \cdots$

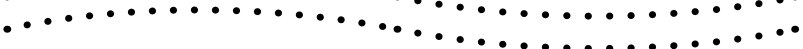

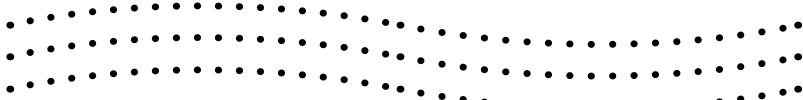
$\ldots \ldots \ldots \ldots \ldots \ldots \ldots \ldots \ldots \ldots \ldots \ldots \ldots \ldots \ldots \ldots$ 